【奨励賞研究】

\title{
Fundamental study and application of decellularized porcine aorta as cardiovascular biomaterials
}

\author{
Pingli Wu \\ Life Science and Technology, Tokyo Medical and Dental University
}

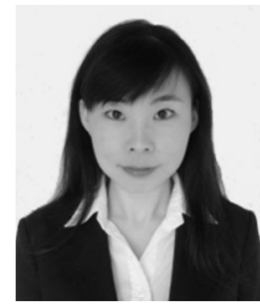

\section{Introduction}

Recently, the decellularized tissues have been widely investigated as scaffolds for regeneration of various tissues and some of them have been developed into products. However, the tissues obtained from animals often possess different size with the human tissues which limited their application. In order to realize the decellularized tissues wide application, we considered to fabricate them into desirable shape and size as other kinds of biomaterials. Decellularized porcine aorta was taken as an example to investigate possibilities of decellularized tissue applied as biomaterials (Fig.1). Properties of tissues vary in the different part of body. Understanding of fundamental properties such as mechanical properties and permeability are very important for its application. Combination with other kind of materials is common method for polymer improvements, which is not been used in decellularized tissues. Therefore, we systemically investigated the fundamental properties of decellularized porcine aorta and investigated their combination possibilities with other kinds of materials.

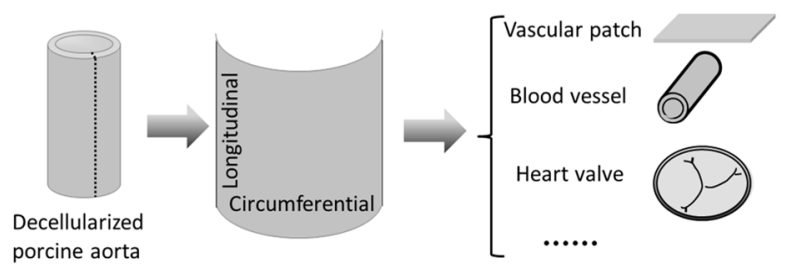

Fig.1 Schematic diagram of decellularization aorta applied as cardiovascular biomaterial

\section{Methods}

The aortic intima-media was peeled form porcine aorta with the thickness of about $0.5 \mathrm{~mm}$. Intima-media was decellularized by sodium dodecyl sulfate (SDS) method ${ }^{1)}$ and high-hydrostatic pressure (HHP) method ${ }^{2}$. The histological structure was characterized by $\mathrm{HE}$ staining and EVG staining. Mechanical properties were evaluated by using tensile strength testing method. BSA and lysozyme were used as model proteins and protein permeability was evaluated by using two-chamber diffusion system. The mass transfer coefficient was calculated according to Fick's law.

HHP decellularized aortic intima-media was combined with electrospun fibers to fabricate small diameter blood vessels. Pressure-diameter relationship and stiffness parameter were used to evaluate their mechanical properties ${ }^{3)}$.

\section{Results and conclusion}

Histological results showed that structure of the HHP treated sample was unchanged histologically, whereas that of the SDS treated sample appeared to be structurally loose. Consequently, the mechanical properties and permeability for an HHP-treated sample was similar to those for an untreated sample, while, the mechanical properties and permeability of SDS decellularized intima-media significantly changed. It was concluded that the structure changes of the decellularized tissue were depended on the decellularization process, and the structure determined the fundamental properties of the decellularized aorta. Different decellularization processes can alter the aorta structure. Two-layered blood vessel with $4 \mathrm{~mm}$ in diameter was prepared by combination of the decellularized porcine aortic intima-media with electrospun fibers. It can be also concluded that decellularized tissues can be combined with synthetic materials. The combination can effectively improve the mechanical properties of decellularized tissue.

\section{Conclusion}

The fundamental properties of decellularized porcine aortic intima-media decellularized by HHP and SDS method was systemically investigated. Fundamental properties changes depended on the decellularization method. The combination of decellularized intima-media showed that the decellularized aorta can be used as a biomaterial and modified by synthetic materials. This concept can broaden the application of decellularized tissue in the future.

\section{Reference}

[1] Korossis SA, Wilcox HE, Watterson KG, Kearney JN, Ingham E, Fisher J. J Heart Valve Dis. 2005; 14(3): 408-421.

[2] Funamoto S, Nam K, Kimura T, et al. Biomaterials. 2010; 31(13): 3590-3595.

[3] Hayashi K, Mori K, Miyazaki H. Am J Physiol Heart Circ. Physiol. 2003; 284 (2): H511-H518. 